\title{
L'enherbement du vignoble alsacien : un bilan positif vis-à-vis du transfert de nitrates
}

Grass in the Alsatian vineyard: A positive assessment with respect to the transfer of nitrate

Gras im elsässischen Weinbau : Eine positive Bilanz gegenüber der Übertragung von Nitrat

\section{Caroline Grégoire et Julien Tournebize}

\section{OpenEdition}

\section{Journals}

Édition électronique

URL : http://journals.openedition.org/rge/1041

ISSN : 2108-6478

\section{Éditeur}

Association des géographes de l'Est

Édition imprimée

Date de publication : 1 janvier 2004

Pagination : 55-65

ISSN : 0035-3213

\section{Référence électronique}

Caroline Grégoire et Julien Tournebize, «L'enherbement du vignoble alsacien : un bilan positif vis-à-vis du transfert de nitrates », Revue Géographique de l'Est [En ligne], vol. 44 / 1-2 | 2004, mis en ligne le 03 juin 2009, consulté le 07 septembre 2020. URL : http://journals.openedition.org/rge/1041

Ce document a été généré automatiquement le 7 septembre 2020.

Tous droits réservés 


\section{L'enherbement du vignoble alsacien : un bilan positif vis-à-vis du transfert de nitrates}

Grass in the Alsatian vineyard: A positive assessment with respect to the transfer of nitrate

Gras im elsässischen Weinbau : Eine positive Bilanz gegenüber der Übertragung von Nitrat

Caroline Grégoire et Julien Tournebize

\section{NOTE DE L'ÉDITEUR}

Article reçu le 19 avril 2004 ; accepté le 24 juin 2004

Nous tenons à remercier le Lycée Agricole et Viticole de Rouffach pour la mise à disposition des parcelles et pour sa constante et fructueuse collaboration dans ce projet.

\section{L'enherbement du vignoble alsacien : un véritable enjeu régional}

1 La dégradation de la qualité de l'eau de la nappe phréatique rhénane alsacienne devient cruciale dans certains secteurs. Depuis les années 1970, on assiste à une augmentation constante des concentrations en nitrates dans les eaux souterraines alsaciennes. L'évolution de la qualité des eaux souterraines est fortement liée à tous les types de cultures pratiquées et à leur conduite. Dans un bassin versant alsacien, la partie supérieure, située sur le piémont vosgien constitue une zone privilégiée pour la recharge des nappes souterraines ; or, elle est souvent occupée par le vignoble qui peut ainsi contribuer à la concentration en nitrate des eaux de nappe. Ce vignoble qui s'étend sur 15000 ha est un milieu aux caractéristiques particulières : un parcellaire 
morcelé, une pente parfois importante (supérieure à $10 \%$ ), une culture en rang pérenne et souvent associée à un enherbement, des petits bassins versants secs sans écoulement permanent, la présence de nombreux chemins et des itinéraires techniques variables forment des conditions favorables à des phénomènes hydrologiques propres, en particulier lors des événements pluvieux intenses. Parmi ces spécificités, nous nous intéressons à la technique d'entretien du sol qui consiste à enherber les inter rangs de vigne : placé tous les inter rangs ou un inter rang sur deux, l'enherbement présente de nombreux avantages dont celui de retenir la pollution nitratée plus particulièrement en période hivernale (rôle de piège à nitrate) comme cela a déjà été montré (Amberger, 1983 ; Ballif, 1994 ; Juergens-Geschwind, 1989).

2 Les objectifs des recherches menées dans le cadre du projet EVA (Enherbement du Vignoble Alsacien) portent sur la détermination, la hiérarchisation et l'évaluation des impacts de cette pratique en comparant les flux hydriques et azotés sous zone racinaire à partir des mesures de terrain réalisées sur deux parcelles, l'une désherbée, l'autre enherbée un rang sur deux. Les résultats acquis intéressent deux campagnes de mesures (1999-2000) calées sur les saisons culturales.

3 L'itinéraire technique choisi et adopté en vignoble alsacien depuis 1975 réside dans l'association de deux plantes au cycle végétatif décalé et aux explorations racinaires différentes : 25 à $40 \mathrm{~cm}$ pour l'herbe (graminées, fétuques), jusqu'à $3 \mathrm{~m}$ pour la vigne (Vitis Vinifera). À l'origine, la fonction de l'enherbement était de limiter les risques d'érosion des sols et plus particulièrement ceux dus au ruissellement. Aujourd'hui, l'enherbement est également considéré comme un moyen de lutte contre la pollution par les nitrates et ce travail du sol est bien introduit dans les pratiques des viticulteurs. Une étude réalisée sur le vignoble haut rhinois et bas rhinois, par télédétection (Image SPOT multispectrale, pixel de $10 \mathrm{~m} \times 10 \mathrm{~m}$ ), a quantifié le taux d'enherbement du vignoble alsacien. Celui-ci a progressé en moyenne de $10 \%$ sur les deux départements entre 1995 et 1999 (dates clés des opérations FERTI-MIEUX « Piémont eau et terroirs » et "Collines eau et terroirs»). En tenant compte des facteurs limitants (stress hydriques, pédologie, ...) qui interdisent la mise en place d'un enherbement même un rang sur deux, $13 \%$ de la surface totale du vignoble restent potentiellement «enherbable». Certaines communes telles que Bernarviller, Molsheim, Mutzig, Nordheim ou Nothalten ont vu le taux d'enherbement de leur vignoble augmenter de plus de $20 \%$ sur 4 ans.

4 Afin de quantifier le fonctionnement global de l'association culturale étudiée, il est nécessaire d'identifier le partage des ressources du milieu entre les deux types de plante. La vigne est prise pour la culture dominante dont la dormance se situe de novembre à mars et l'herbe est considérée comme plante associée de couverture maximale en automne/hiver et dont la gestion maitrisée est pluri-annuelle.

5 Notons que la vigne reste peu étudiée par rapport aux grandes cultures céréalières et l'est souvent dans une perspective physiologique comme le présentent différents auteurs dans les vignobles d'Anjou (Morlat, 1987), dans le bordelais (Pouget, 1984), dans le sud (Trambouze et al., 1998 ; Igounet, 1997), en région champenoise (Ballif, 1999) ou bien encore en vignoble alsacien (Sossi, 1993). En dehors des études sur les interactions sur la santé de la vigne (Cazet, 1997 ; Lebon, 1993 ; Morlat, 1987 ; Moulis, 1994) et sur une prise en compte dans la lutte contre l'érosion hydrique des sols cultivés (Léonard, 2003), l'association vigne/herbe ne fait alors l'objet que de peu de publications dans une démarche environnementale quantitative. 


\section{Le site expérimental de Rouffach}

\section{A. Présentation et caractérisation du site}

6 Le site expérimental se situe sur le domaine du Lycée agricole et viticole de Rouffach (Haut-Rhin), à $15 \mathrm{~km}$ au sud/sud ouest de Colmar (Fig. 1). Le climat sous influence continentale, se caractérise par les paramètres moyens interannuels suivants (calculés sur la période 1946-1998) : pluviométrie : $586 \mathrm{~mm}$, température : $11^{\circ} \mathrm{C}$, ETP PenmanMonteith : $762 \mathrm{~mm}$. Les parcelles expérimentales (de 24 et 42 ares) appartiennent à un bassin versant de 61 ha, sur massif géologique würmien. Elles ont une pente de $14 \%$ en moyenne, orientée Sud-Est. La toposéquence du site correspond à une formation de limon calcaire éolien. Le sol est un sol brun calcaire peu évolué profond (Calcosol), (FOA-UNESCO, 1981). Une caractérisation spécifique du sol a été élaborée pour les deux parcelles expérimentales. Un résumé des principaux résultats est présenté au tableau 1. Des profils racinaires complètent cette caractérisation.

Figure 1 : Site expérimental de Rouffach, Bas-Rhin, France. Limites du bassin versant topographique et localisation des parcelles expérimentales.

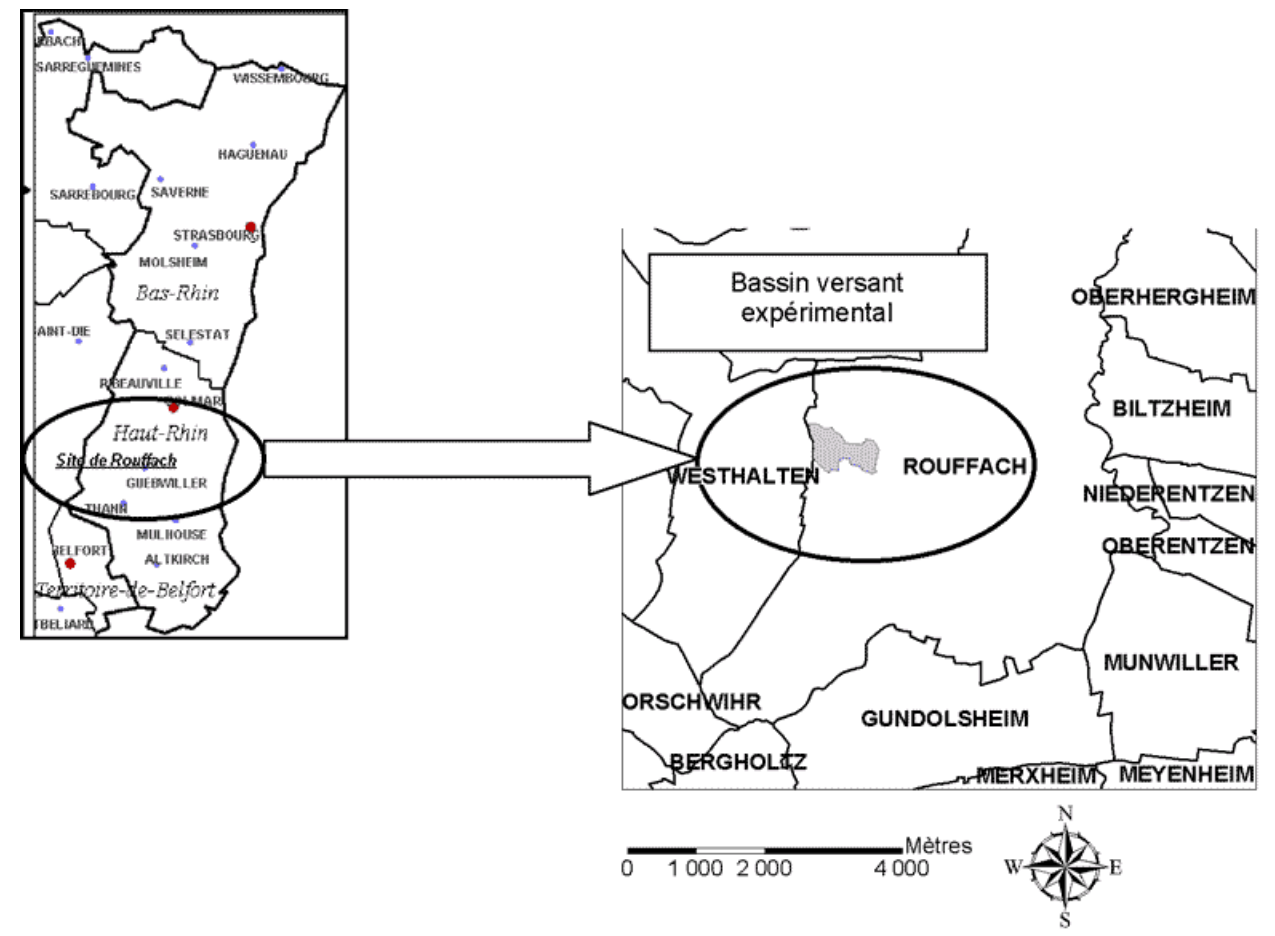

Tableau 1 :Caractérisation des sols des parcelles expérimentales du site de Rouffach (67, France).

\begin{tabular}{|l|l|l|}
\hline & Parcelle enherbée $1 / 2$ & Parcelle témoin désherbée \\
\hline Classe de texture & Limon Argileux & Limon Argileux \\
\hline Matière organique $(\mathrm{g} / \mathrm{kg})$ & 15 & 13 \\
\hline
\end{tabular}




\begin{tabular}{|l|l|l|}
\hline Azote total (mg/g de sol sec) & 1 & 0,9 \\
\hline Dont Azote minéral (\% de l'azote total) & 0,9 & 0,6 \\
\hline C/N & 8,8 & 8,7 \\
\hline RU sur 1 m (mm) & $225( \pm 19)$ & $173( \pm 9)$ \\
\hline Conductivité hydraulique à saturation & & \\
\hline Topsoil (cm/s) & $157( \pm 57)$ & $66( \pm 15)$ \\
\hline Subsoil (cm/s) & $43( \pm 21)$ & $60( \pm 17)$ \\
\hline Profil racinaire & Comptage racinaire & Comptage racinaire \\
\hline PMAX (cm) & 50 & 10 \\
\hline P10 (cm) & 60 & 20 \\
\hline
\end{tabular}

\section{B. Techniques de conduite de la vigne et de l'enherbement}

7 Le cépage des vignes est différent d'une parcelle expérimentale à l'autre: Riesling (plantation 1954) et Tokay-Pinot Gris (plantation 1982), respectivement sur la parcelle désherbée et la parcelle enherbée, sur porte greffe SO4. Le mode de conduite est traditionnel: palissage plan vertical guyot double arcure. La densité de plantation moyenne est de 5000 pieds/ha. L'écartement entre rangs de vigne vaut 1,20 et $1,40 \mathrm{~m}$ respectivement pour les parcelles désherbée et enherbée. La parcelle désherbée ne subit aucun travail du sol tandis que la parcelle enherbée nécessite l'entretien du couvert herbacé par plusieurs fauches. La fertilisation des parcelles a diminué de 60 kgN/ha en 1995 à $22 \mathrm{kgN} / \mathrm{ha}$ de 1996 à 1998 puis $0 \mathrm{kgN} / \mathrm{h}$ a de 1999 à 2000.

8 Malgré la différence de cépage, d'entretien du sol, les comportements spécifiques de la vigne (croissance, rendement,...) seront considérés comme identiques dans la suite du travail.

9 L'enherbement de la parcelle enherbée un rang sur deux est défini comme naturel maîtrisé. Il est constitué principalement de ray-grass, de brome stérile, et les adventices classiques dépendant de la saison (géranium, séneçon, mercuriales, amaranthe, ...).

\section{Profils racinaires}

10 Le nombre de racines de tout diamètre est compté par horizon, sur une largeur de $100 \mathrm{~cm}$ et tous les $10 \mathrm{~cm}$ à partir de la surface. Ces valeurs absolues sont transformées en fréquence (\%) de racines dans chaque horizon de $10 \mathrm{~cm}$ par rapport au nombre total de racines observé dans l'ensemble du profil. Nous pouvons ainsi calculer PMAX (profondeur correspondant au maximum de densité, qui indique l'horizon le plus 
favorable à la prospection racinaire) et P10 (profondeur correspondant à la densité inférieure à 10 \% qui marque une forte diminution de la prospection racinaire).

\section{Instrumentation}

11 L'acquisition des mesures s'est déroulée durant les saisons culturales 1999 et 2000.

12 Le suivi climatique est assuré par une station météorologique à 5 paramètres de type MIRIA5 placée à 250 mètres à vol d'oiseau des parcelles expérimentales. Celles-ci sont équipées de quatre stations de mesure, constituant 4 répétitions le long d'un rang de $100 \mathrm{~m}$. Chaque station (rang et inter-rang) comprend une batterie de tensiomètres (cannes et capteur de pression SMS 2500S, SDEC), bougies poreuses (diamètre $63 \mathrm{~mm}$, SDEC), sondes de température (PT100, Mesurex) et tubes d'accès TDR (TRIME, IMKO) à quatre profondeurs $30,60,90,120$. Des pièges à ruissellement complètent cette instrumentation.

13 Les analyses de nitrate, ammoniac de l'eau prélevée dans le sol et de l'eau de pluie sont effectuées sur un analyseur en flux continu de type Alliance au Laboratoire d'Études des Eaux de l'ENGEES, Strasbourg.

Les mesures sont effectuées à un pas de temps hebdomadaire. Dans ce travail, seuls les résultats saisonniers, résultant du suivi hebdomadaire, sont interprétés afin d'élaborer les bilans de masse nécessaires à la compréhension des effets environnementaux de l'enherbement sur la culture de la vigne.

\section{E. Méthode de calcul du flux hydrique sous la zone racinaire de la vigne}

15 La méthode consiste à estimer le flux hydrique en dessous de la couche considérée par hypothèse " hors de la zone racinaire active », en utilisant la loi de Darcy. Le principe du calcul des flux hydriques infiltrés (FI) associe le gradient hydraulique pour déterminer le sens des écoulements, à la variation de stock. L'estimation des variations de stocks permet de quantifier les volumes infiltrés en faisant l'hypothèse simplificatrice que la variation de stock entre deux dates, quand l'écoulement est descendant, est seulement la résultante de l'infiltration profonde. Une estimation quantitative de l'infiltration profonde (FI) au pas de temps hebdomadaire s'exprime de la façon suivante par:

$$
\vec{q}=-K \cdot \vec{\nabla} h \text { soit } q=-K \cdot \frac{\Delta \mathrm{h}}{\Delta \mathrm{z}}
$$

pour le sens de l'écoulement et

$$
F I=\frac{\mathrm{q}}{\mathrm{qq} \mid} \cdot \Delta S_{n}^{2}
$$

avec $\vec{q}$ le flux de Darcy, $S_{\mathrm{t} 1}^{\mathrm{t}}$ la variation de stock d'eau entre deux dates t1 et t2, K le tenseur de conductivité hydraulique, h la charge hydraulique, $\mathrm{z}$ la côte. Nous pouvons alors en déduire la quantité lessivée d'azote nitrique (Lixiv) comme le produit

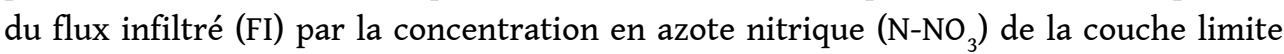
estimée par les profils racinaires à $90-120 \mathrm{~cm}$ : 


\section{Lixiv $=\overline{\mathrm{FI} \cdot\left[\mathrm{N}-\mathrm{NO}_{3}\right]}$}

\section{Résultats}

17 Les mesures ponctuelles et le suivi sur deux ans ont permis de mettre en évidence des comportements contrastés des deux types de parcelles étudiées. Les premiers résultats présentent les effets sur la structure du sol, nous montrons ensuite l'effet induit par la présence d'un deuxième couvert végétal (l'herbe) sur la dynamique du transfert de masse (eau et nitrate).

\section{A. Analyse des sols}

18 Au niveau des caractéristiques du sol, nous constatons une légère augmentation de la matière organique dans l'horizon de surface en parcelle enherbée. En analysant la répartition de l'azote sous toutes ses formes (i.e. organique et minérale), nous concluons à un échange de la forme principale minérale (nitrate) en la forme organique estimée indirectement par la mesure de la matière organique. Nous pouvons aussi distinguer la parcelle désherbée de la parcelle enherbée par le biais des conductivités hydrauliques à saturation: un ordre de grandeur sépare les mesures. Nous en concluons que l'aération y est ainsi mieux assurée. Ceci est confirmé par une réserve utile (RU) plus importante sur le profil en parcelle enherbée $(225 \mathrm{~mm})$ qu'en parcelle désherbée $(173 \mathrm{~mm})$, prouvant un porosité supérieure.

L'analyse des profils racinaires montre que la zone racinaire active (permettant la vie et la croissance de la vigne) se situe dans les deux cas dans le premier mètre du sol. Ainsi, il est légitime de considérer la profondeur de $120 \mathrm{~cm}$ comme profondeur sous racinaire en dessous de laquelle les échanges vigne/sol n'ont plus lieu. De plus le pic de racine (PMAX) de la vigne sous parcelle enherbée $(50 \mathrm{~cm})$ est supérieur à celui de la parcelle désherbée $(10 \mathrm{~cm})$, montrant ainsi un approfondissement de l'architecture racinaire (Morlat, 1981).

\section{B. Analyse hydrique}

L'analyse climatique montre un cumul pluviométrique exceptionnellement important sur les deux années de suivi. Après une pluviométrie normale en 1998 avec $578 \mathrm{~mm}$ (586 mm, moyenne sur 40 ans), les deux années de mesure présentent une pluviométrie de 712 et $703 \mathrm{~mm}$ respectivement pour 1998/1999 et 1999/2000, ce qui correspond à une période de retour supérieur à 70 ans. Ceci a pour conséquence d'augmenter les excédants hydriques et ainsi de limiter les risques de concurrence hydrique pendant la durée du suivi.

21 Au niveau du ruissellement, les effets de l'enherbement se distinguent selon la saison: limitation de l'effet des pluies orageuses (efficacité), et sans effets pour les ruissellements hivernaux. Sur les deux campagnes, nous avons mesuré une diminution des volumes ruisselés de $50 \%$ sous parcelle enherbée.

$\mathrm{Au}$ niveau de l'infiltration profonde, les volumes calculés s'élèvent à $112 \mathrm{~mm}$ en parcelle désherbée et à $70 \mathrm{~mm}$ en parcelle enherbée un rang sur deux, pour 1998/1999. L'écart entre les deux itinéraires techniques diminue la deuxième année (125 mm en 
parcelle désherbée contre $107 \mathrm{~mm}$ en parcelle enherbée un rang sur deux), vraisemblablement dû à la succession des pluviométries exceptionnelles. La grande dispersion des mesures d'humidité implique une grande variabilité des lames drainantes calculées, d'où un écart-type très important.

La différence dans les flux infiltrés est attribuée à l'augmentation des pertes atmosphériques par évapotranspiration du couvert herbacé, estimée à $75 \mathrm{~mm}$. Une tendance générale se détache de l'étude du tableau 2 : l'enherbement diminue d'au moins 30 \% la lame drainée.

Tableau 2 : Lames drainantes calculées à partir des variations de teneur en eau et des gradients hydrauliques en parcelles désherbée et enherbée $1 / 2$, site de Rouffach (68, France).

Campagne 1 : du 01/10/1998 au 30/09/1999; campagne 2 : du 01/10/1999 au 30/09/2000.

\begin{tabular}{|l|l|l|l|l|}
\hline & $\begin{array}{l}\text { Parcelle } \\
\text { désherbée }\end{array}$ & $\begin{array}{l}\text { Parcelle } \\
\text { enherbée 1/2 }\end{array}$ & \\
\hline Unité mm & Campagne 1 & Campagne 2 & Campagne 1 & Campagne 2 \\
\hline Pluviométrie Météo France & 712,8 & 703,2 & 712,8 & 703,2 \\
\hline ETP Météo France & 724,7 & 750,2 & 724,7 & 750,2 \\
\hline Mesure du Ruissellement & 14,1 & 18,8 & 7 & 9,4 \\
\hline $\begin{array}{l}\text { Estimation du Flux Infiltration } \\
\text { profonde (FI) }\end{array}$ & $112,1(48)$ & $125,6(46)$ & $70,3(18)$ & $107,4(35)$ \\
\hline $\begin{array}{l}\text { Rapport FI entre parcelle désherbée } \\
\text { et enherbée }\end{array}$ & $\begin{array}{l}\text { Campagne 1 } \\
\text { Campagne 2 }\end{array}$ & & $\begin{array}{l}63 \% \\
85 \%\end{array}$ \\
\hline
\end{tabular}

\section{Analyse de l'azote}

24 L'ordre de grandeur des quantités exportées par le lessivage s'élève à une vingtaine de kilogrammes d'azote par hectare en parcelle désherbée, et à quelques kilogrammes en parcelle enherbée un rang sur deux (tableau 3). La différence est marquée malgré de grands écart-types (70\%). L'écart-type sur la valeur calculée s'explique par les grandes variabilités des concentrations en nitrate du sol mesurées et de la valeur calculée de l'infiltration.

Tableau 3 : Lessivages calculés à partir de l'estimation des flux infiltrés et de la concentration mesurée pour la couche $90-120 \mathrm{~cm}$ en parcelles désherbée et enherbée $1 / 2$, site de Rouffach (68, France).

Campagne 1 : du 01/10/1998 au 30/09/1999; campagne 2 : du 01/10/1999 au 30/09/2000.

\begin{tabular}{|l|l|l|l|l|}
\hline & \multicolumn{2}{|l|}{ Parcelle désherbée } & \multicolumn{2}{l|}{ Parcelle enherbée 1/2 } \\
\hline Unité $\mathrm{kgN}^{2} \mathrm{NO}_{3} / \mathrm{ha}$ & Campagne 1 & Campagne 2 & Campagne 1 & Campagne 2 \\
\hline Mesure fertilisation & 22 & 0 & 22 & 0 \\
\hline
\end{tabular}




\begin{tabular}{|l|l|l|l|l|}
\hline Estimation variation de stock & $-100(25)$ & $-1(5)$ & $+10(3)$ & $-35(20)$ \\
\hline Estimation du lessivage (Lixiv) & $37,6(23)$ & $34,8(19)$ & $4,4(2)$ & $10,2(7)$ \\
\hline Rapport Lixiv & Campagne 1 & $12 \%$ & \\
& Campagne 2 & $0 \%$ & \\
\hline
\end{tabular}

Une grande variation de stock s'est produite sous parcelle désherbée, témoignant du phénomène de lessivage de stock principalement causé par un fort excédent hydrique. Ce phénomène a lieu la deuxième année en parcelle enherbée mais de façon limitée.

\section{L'enherbement : un bilan globalement positif pour l'environnement}

\section{A. Effets de l'enherbement sur l'architecture racinaire}

26 Le comportement du sol enherbé est modifié par la présence du couvert par rapport à un sol désherbé : l'herbe joue un rôle direct dans les trente premiers centimètres du sol. La conduite de l'enherbement induit une modification de la structure du sol et plus particulièrement du système racinaire de la vigne dans le sens d'un approfondissement de celui-ci pour pallier la concurrence hydrique provoquée par l'enherbement. Cette nouvelle géométrie du système de prélèvement racinaire de la vigne montre une grande capacité d'adaptation de celle-ci à son environnement.

\section{B. Effets de l'enherbement sur le comportement hydrique des sols}

27 Le comportement hydrique des sols est différent selon que l'on pratique un enherbement ou pas. Le sol nu exposé aux aléas climatiques présente une croûte de battance s'opposant à l'infiltration et présente une macroporosité ainsi que des chemins préférentiels (e.g. fissuration) accélérant l'infiltration. Le ruissellement, supérieur en parcelle désherbée, provoque un léger déficit d'infiltration lors des épisodes orageux estivaux. Ceci a pour effet de provoquer un dessèchement de la couche de surface si l'orage est suivi d'une longue période sèche. La limitation de la perte en eau par ruissellement est cependant restreinte et ne concerne que quelques dizaines de millimètres, insuffisants pour combler le déficit généré par le prélèvement hydrique de l'herbe.

28 La présence d'herbe modifie le régime hydrique des parcelles au cours des différentes saisons : en période humide, le sol enherbé a une plus grande capacité à stocker l'eau, la réserve utile étant supérieure du fait de l'augmentation de la bioporosité (porosité due à la présence de racines) du sol. Lors de périodes sèches (en général en été), l'herbe étant plus sensible que la vigne au dessèchement, le couvert prend l'aspect d'un paillasson jaune (type mulch) qui protège le sol de l'évaporation et du ruissellement, ainsi seule la vigne prélève l'eau du sol.

L'enherbement modifie les mécanismes de redistribution de l'eau infiltrée (disponibilité de l'eau, ruissellement et drainage naturel). L'herbe, en augmentant la 
bioporosité du sol, augmente la capacité de rétention en eau mais elle accentue les phénomènes d'assèchement du sol.

30 Le déficit engendré par l'évapotranspiration de l'herbe se répercute aussi sur les volumes d'eau drainée.

31 L'enherbement un rang sur deux, dans le contexte incluant une année humide, induit une réduction du drainage naturel de 30 à $40 \%$. L'ordre de grandeur des volumes mesurés et estimés s'élève à $100 \mathrm{~mm}$ en parcelle désherbée et à $70 \mathrm{~mm}$ en parcelle enherbée, pour une année exceptionnellement pluvieuse.

Soulignons que nos résultats sont en accord avec les travaux précédents (Gaildraud, 1996) : le drainage naturel est prépondérant sur le ruissellement. Il représente plus de quatre fois le ruissellement, en parcelle désherbée.

\section{Effets de l'enherbement vis-à-vis des transferts de nitrates}

En ce qui concerne le ruissellement, l'enherbement augmente la concentration en azote total dans les volumes ruisselés, tout en restant inférieure à $15 \mathrm{mg} / \mathrm{L}$ de $\mathrm{NO}_{3}$. Au niveau des flux exportés, même si les quantités restent marginales, l'enherbement est efficace au regard des volumes ruisselés réduits.

34 Le lessivage de nitrate doit rester la cible privilégiée de la démonstration: malgré l'imprécision des mesures de concentration liée à la grande variabilité naturelle du sol, que ce soit avec ou sans fertilisation raisonnée, la moyenne des concentrations mesurées dans la solution du sol en parcelle enherbée se situe en dessous de la valeur seuil de $50 \mathrm{mg} / \mathrm{L}$ de nitrates, limite de potabilité, alors qu'en parcelle désherbée, elle est bien au dessus (entre 50 et $500 \mathrm{mg} / \mathrm{L}$ ).

En parcelle désherbée, en 1998, les stocks d'azote nitrique sont estimés à 300 kgN-NO ${ }_{3} /$ ha, assurant largement les besoins de la vigne. La fertilisation, même réduite, était alors superflue. Néanmoins, nous pouvons remarquer que les stocks de nitrate du sol ont fortement diminué à partir de mai 1999, jusqu'à un stock moyen de $100 \mathrm{kgN}-\mathrm{NO}_{3} / \mathrm{ha}$, montrant ainsi la rapide réactivité du sol, en conditions climatiques favorables, à la lixiviation et en moindre importance à la dénitrification.

En parcelle enherbée, un équilibre azoté semble atteint entre les quantités prélevées par l'association vigne/

herbe et les quantités apportées par la pluie et minéralisées naturellement par le sol. Notons que l'apport de fertilisant, en 1998, a été absorbé par le couvert herbeux sans bénéfice pour la vigne. Ceci montre l'effet tampon de la couche enherbée vis-à-vis de la fertilisation et de tout excès de nitrate, pouvant à terme être lessivé.

Les flux calculés sur la base de la seule forme nitrique de l'azote montrent, pour la période observée, une réduction du lessivage en nitrate d'un facteur $7\left(34 \mathrm{kgN}-\mathrm{NO}_{3} / \mathrm{ha}\right.$ en parcelle désherbée contre moins de $5 \mathrm{kgN}-\mathrm{NO}_{3} /$ ha en parcelle enherbée un rang sur deux, pour la campagne 1) entre parcelle désherbée et enherbée. La mise en herbe des inter rangs de vigne, quand il est possible, semble donc, sur la base de ces deux campagnes de mesure, participer à la protection des eaux d'infiltration. L'enherbement permet de contenir et maîtriser les stocks d'azote nitrique potentiellement lessivables. Cependant l'enherbement a pour principal impact le changement dans la dynamique de l'azote. En effet l'azote minéral du sol, disponible en parcelle désherbée est immobilisé sous forme organique par la biomasse du couvert herbacé. C'est dans ce sens que nous 
nuancerons la notion de piège à nitrate et préférerons celle d'immobilisateur d'azote. Ceci laisse à supposer que lors du retournement de sol pour une rotation de culture, en fin de cycle de vie de la vigne, qui a lieu tous les trente à quarante ans en général, un pic de minéralisation s'observera en parcelle enherbée. Le problème que pose l'enherbement réside alors dans la continuité de cet itinéraire technique : que se passe t'il après l'enherbement ?

\section{Conclusion et perspectives}

Les pertes sous la zone racinaire et à terme vers les eaux souterraines des matières fertilisantes azotées peuvent être réduites en zone de vignoble grâce à la pratique de l'enherbement. Cet itinéraire technique, conduit de façon raisonnée, induit plusieurs effets tant sur le sol que sur la vigne elle-même et son environnement. Les mesures réalisées pendant deux ans au pas de temps hebdomadaires et présentés ici sous forme de bilans saisonniers, sous régimes hydriques différents, traduisent cependant des phénomènes installés puisqu'aucun stress hydrique n'a été constaté sur les années de suivi.

Après quelques années d'instauration, la conduite de l'enherbement sur une parcelle de vigne induit donc une modification du cycle de l'eau en augmentant les retours vers l'atmosphère et en diminuant les infiltrations profondes de $70 \mathrm{~mm} / \mathrm{an}$ par rapport à une parcelle non enherbée. En ce qui concerne l'azote, le calcul du lessivage effectué sur la forme nitrique montrent un taux moindre sous parcelle enherbée (réduction de plus de $50 \%$ pour une parcelle enherbée un rang sur deux par rapport à une parcelle désherbée). Il faut cependant rester prudent en ce qui concerne ces résultats car l'azote se trouve alors mobilisée sous forme organique.

En conclusion, nous pouvons dire que l'herbe n'est pas un piège à nitrate mais un immobilisateur d'azote sous forme organique. Si l'enherbement est mal géré, la quantité d'azote en rétention dans le sol peut être à nouveau mobilisée et être lessivée. La gestion de cet itinéraire technique doit donc être menée à long terme.

41 Le facteur principal de limitation de l'enherbement n'est pas la quantité de nitrate du sol disponible mais bien la quantité d'eau disponible contrôlée par la pluviométrie et c'est d'ailleurs bien ce facteur qui conduit les viticulteurs à enherber ou pas dans une perspective de préservation des rendements.

Plusieurs études (Grégoire, 1999; Alajaouine, 2001) ont conduit à évaluer le taux d'enherbement du vignoble alsacien par télédétection : en 1999, plus des trois quarts du territoire viticole était alors soit enherbé un inter rang sur deux, soit tous les inter rangs. Au regard du stress hydrique induit par une année sèche et/ou un sol filtrant, qui conduit à la baisse des rendements et interdit l'enherbement, (Girault, 2001 et Mailhe, 2002) ont ensuite montré que $13 \%$ du vignoble était encore potentiellement «enherbable». C'est sur ces zones ainsi ciblées que doit alors se porter l'effort des viticulteurs accompagnés par les conseillers agricoles.

Rappelons enfin que cette première approche reste dépendante des mesures effectuées, de leur durée et fréquence d'acquisition. Une des perspectives de ce travail s'oriente vers la modélisation qui permettra de simuler des évolutions journalières des transferts de la pollution nitratée sous parcelles (modélisation à base physique) et à l'échelle du 
bassin versant (modélisation semi-spatialisée basée sur la description des unités fonctionnelles et la caractérisation des liaisons et interactions entre elles).

\section{BIBLIOGRAPHIE}

ALAJOUANINE A. (2001). - Exploitation d'images satellites SPOT pour le suivi de l'enherbement en zone viticole : application au projet EVA (Enherbement du Vignoble Alsacien). Mémoire de fin d'Études, ENSAIS, ENGEES, Strasbourg. Deux tomes, 143 p.

AMBERGER A. (1983). - Ways to control the availability, turnover and losses of mineral fertilizer N in soils. Economic Commission for Europ, Committe on Agricultural Problems, Food and Agriculture Organization of the United Nations, Symposium on Research into Agrotechnical methods aiming at increasing the productivity of crops, Geneva, January 17-21, pp. 1-25.

BALLIF J.L. (1994). - Lysimètres en monolithes d'une rendzine brune sur craie cryoturbée. Bilans hydrique et minéral en sols nu, enherbé, cultivé et planté en vigne 1973-1974 à 1990-1991, INRA, Chalons-en-Champagne, $124 \mathrm{p}$.

BALLIF J.L. (1999). - Ruissellement et érosion en Champagne sur sols de vignes et de cultures : observations, mesures, prévisions et remèdes, Ed. Johanet, Paris, 158 p.

CAZET S. (1997). - Contribution à l'élaboration d'une carte conseil à l'enherbement permanent des sols viticoles champenois, mémoire de DEA, Institut National Agronomique et CIVC, Epernay, $55 \mathrm{p}$.

Curt T., Bouchaud M., Lucot E.C., BARDonnet L. et Bouquet F. (1998). - Influence des conditions géopédologiques sur le système racinaire et la croissance en hauteur du Douglas dans les monts du Beaujolais. Ingénieries - EAT 16, pp. 29-46.

FAO-UNESCO (1981). - Soil map of the world. 1/5,000,000. Food and Agricultural Organisation, Roma.

GAILDRAUD C. (1996). - Étude de l'impact du ruissellement dans le vignoble sur la qualité de la nappe phréatique d'Alsace. Azote, phosphore, sulfates et produits phytosanitaires. Août 1990 à décembre 1995, DIREN-SEMA \& ARAA, Strasbourg, 65 p.

Girault A. (2001). - Le vignoble alsacien. Sensibilité à l'infiltration hydrique verticale. Risque de stress hydrique généré sur la vigne par la mise ne place d'un couvert herbacé. Trois tomes (Mémoire, annexes, atlas cartographique), $63 \mathrm{p}$.

GREGoIRE C. (1999). - L'enherbement des vignes, un geste simple pour l'environnement : le cas alsacien. L'eau, l'industrie, les nuisances, mai, n 22, pp. 24-30.

IGOUNET O. (1997). - Modifications des états de surface des sols viticoles par paillages artificiels et par enherbement : incidences sur le microclimat de la vigne et la conservation des sols. Thèse, Université Louis Pasteur - U.F.R. de Géographie, 147 p.

JUERGENS-GESCHWIND S. (1989). - Ground water nitrates in other developed countries (Europe). Relationships to land use patterns. In : « Nitrogen management and ground water protection », R.F. FolLETT. Developments in agricultural and managed-forest ecology, $\mathrm{n}^{\circ} 21$, pp. 73-138. 
LEONARD M. (2003). - L'érosion hydrique des sols cultivés : analyse systémique et proposition de gestion. Application aux vignobles d'Ardèche méridionale. Thèse de l'Université Joseph Fourrier (Grenoble I). Deux tomes, $561 \mathrm{p}$.

LEBON E. (1993). - De l'influence des facteurs pédo- et mésoclimatiques sur le comportement de la vigne et les caractéristiques du raisin. Thèse, Université de Bourgogne, $165 \mathrm{p}$.

MAILHE A. (2002). - Élaboration d'indicateurs viti-environnementaux pour une meilleure gestion du territoire, projet Enherbement du Vignoble Alsacien. Mémoire de fin d'études ENGEES, 100 p.

MoRLat R. (1981). - Effets comparés de deux techniques d'entretien du sol sur l'enracinement de la vigne et sur le milieu édaphique. Agronomie, 1 (10), pp. 887-896.

Morlat R. (1987). - Influence du mode d'entretien du sol sur l'alimentation en eau de la vigne en Anjou. Conséquences agronomiques. Agronomie, 7 (3), pp. 183-191.

Moulis I. (1994). - L'enherbement de vignobles méditerranéens : importance de la compétition hydrique vigne/culture intercalaire herbacée en vue d'une maitrise de la production viticole, ENSAM, Montpellier, 100 p. + annexes.

POUGET R. (1984). - Action de la concentration de la solution nutritive sur quelques caractéristiques physiologiques et technologiques chez Vitis vinifera L. cv. « CabernetSauvignon ». 1. Vigueur, rendement, qualité du moût et du vin. Agronomie, 4 (5), pp. 437-442.

Sossi L.- Opération Ferti Mieux sur le Piémont Haut-Rhinois. Chambre Agriculture 68, Colmar, 36 p. TRAMBOUZE W., BERTUZZI P. et VOLTZ M. (1998). - Comparison of methods for estimating actual evapotranspiration in a row-cropped vineyard. Agricultural and Forest Meteorology, $\mathrm{n}^{\circ}$ 91, pp. 193-208.

\section{RÉSUMÉS}

La pratique culturale consistant à associer deux cultures au cycle végétatif déphasé : la vigne (Vitis Vinifera) et l'herbe (graminées, fétuques) est évaluée. Le suivi sur deux saisons de deux parcelles (l'une enherbée un rang sur deux, l'autre désherbée) montre l'influence de l'herbe sur la dynamique du cycle de l'eau et des nitrates. Sur les deux campagnes de mesure (1998/1999 et 1999/2000), on constate une perte hydrique supplémentaire de $70 \mathrm{~mm} / \mathrm{an}$ par évapotranspiration sur parcelle enherbée un rang sur deux. La présence d'herbe tamponne la fertilisation, mobilise l'azote sous forme organique et non plus minérale et provoque une réduction des flux d'azote jusqu'à un facteur 7.

Cultivation method consisting in associating two row cultures with a vegetative cycle out of phase: the vineyard and the grass is evaluated. The two years follow-up of two plots (one with grass each other row, the other weeded) shows the impact of the grass on the dynamics of the water and nitrate cycle. During the two series of measurement (1998/1999 and 1999/2000), we note an additional loss of $70 \mathrm{~mm} /$ an per evapotranspiration on the plot with grass. The presence of grass also regularizes any fertilization

and mobilizes the nitrogen in a organic form and either mineral form. The presence of grass on a plot causes a reduction of nitrogen flows until a factor 7 .

Es wird die Anbaupraxis bewertet, die zwei kulturen mit phasenlosem Vegetationszyklus verbindet: die Weinrebe (Vitis Vinifera) und das Gras (Gramineen, Festuken). Die jährlich zweimalige Fruchtfolge auf zwei Parzellen, die eine mit, die andere ohne Gras, zeigt den Einfluß des Grases auf die Dynamik des Wasserzyklus und der Nitrate. Es wird ein zusätzlicher hydrischer 
Verlust von $70 \mathrm{~mm} / \mathrm{Jahr}$ durch Evatranspiration auf begraster Parzelle festgestellt. Die Mitwirkung von Gras puffert die Fruchtbarmachung, mobilisiert den Stickstoff in organischer und nicht mehr mineralischer Form und ruft eine Reduktion des Stickstoffflusses bis zum Faktor 7 hervor.

INDEX

Schlüsselwörter : Begrasung, Infiltration, Nitrate, Weinrebe

Keywords : grass, infiltration, nitrate, runoff, vineyard

Mots-clés : enherbement, infiltration, nitrate, ruissellement, vigne

\section{AUTEURS}

\section{CAROLINE GRÉGOIRE}

Centre d'Écologie Végétale et d'Hydrologie (CEVH) de l'ENGEES, 1 quai Koch, BP 61039, F-67070 Strasbourg Cedex, France, caro@engees.u-strasbg.fr

\section{JULIEN TOURNEBIZE}

UR Ouvrages pour le Drainage et l'Étanchéité, Cemagref, Parc de Tourvoie, F-92163 Antony, France 